\title{
Best Agers - Altern in bestmöglicher Gesundheit
}

„Best Agers“ - das Thema dieses Schwerpunktes lässt sich in englischer Sprache kurz und prägnant ausdrücken. „Altern in bestmöglicher Gesundheit, das Beste aus dem Älterwerden machen". Alt an Jahren, aber mit einem jung gebliebenen Geist in einem gesunden Körper das Lebensende zu erreichen, wie gelangt man an dieses Ziel? Ab wann zählen wir uns denn zu den „Älteren“? Ab dem Zeitpunkt des Rentenantritts (wenn wir in Österreich in Pension gehen), in Deutschland derzeit mit knapp 66 Jahren? Wenn wir in den Status der Großeltern aufsteigen? Wenn wir durch das Auftreten einer schweren Krankheit merken, dass wir sterblich sind?

Die WHO definiert mehrere Stadien des „Altseins“: Zwischen 65 und 80 Jahren gilt man als „old“, von 80 bis 100 als „oldest-old“ und ab 100 als „Centenarian“. 1982 unterzeichneten die Staaten der UNO in Wien ein Dokument, das als Vienna International Plan of Action on Ageing (IPAA) bezeichnet wird und zum Ziel hat, „die Kapazitäten der Regierun-

Deutsche Zeitschrift für Akupunktur 2021 • 64 (4): 270-271

https://doi.org/10.1007/s42212-021-00422-4

Angenommen: 21. September 2021

(c) Springer Medizin Verlag $\mathrm{GmbH}$, ein Teil von

Springer Nature 2021 gen und der Zivilgesellschaft zu stärken, um wirksam mit der Alterung der Bevölkerung umzugehen ..." [1]. 2050 werden gemäß den Angaben der WHO die über 65-Jährigen einen Anteil von 16,4 \% der Gesamtbevölkerung stellen, statt 135.000 im Jahr 2000 wird es 2050 voraussichtlich mehr als 2 Mio. über 100-Jährige geben.

\section{I) Die WHO definiert mehrere Stadien des "Altseins"}

Eines der Hauptthemen des Welt-Gesundheitstages am 07.04.1999 befasste sich mit den vielfältigen Herausforderungen einer veränderten Altersstruktur. Dr. Gro Harlem Brundtland, damalige Generaldirektorin der WHO, brachte es auf den Punkt: „Active ageing makes the difference" [1].

Aktives Altern impliziert Bewegung, und so sei zunächst auf den Beitrag von Herrn Univ. Prof. Dr. med. Dr. phil. Winfried Banzer hingewiesen. Eine wichtige ärztliche Aufgabe stellt für den (Sport-) Mediziner die Bewegungsberatung dar, diese sollte in keinem ärztlichen Gespräch fehlen.
Die chinesische Medizin schätzt seit Jahrtausenden die sanften fließenden Bewegungsabläufe zur Lebenspflege. Frau Dr. Ute Engelhardt weist in ihrem Artikel auf die vielfältigen gesundheitlichen Einflüsse von Qigong und Tai-Chi hin, untermauert von klassischen Texten und neuesten klinischen Erkenntnissen.

\section{》) Aktives Altern impliziert Bewegung}

Ernährung nach den „5 Elementen (Phasen)“, das Wissen um Lebensmittel als „Mittel zur Lebensverlängerung“, entstammt ebenfalls der Tradition. Gestützt auf fundiertes Wissen zu Mikronährstoffen, Kalorienaufnahme, Mikrobiom etc. gibt Frau Dr. Alexandra Knauer Ernährungsempfehlungen inklusive eines Rezepts zur Stärkung der „Mitte“.

Um Ernährung geht es unter anderem auch im Beitrag von Leonhard Kostner, der seine Erkenntnisse aus einer schulischen Recherche diesem Schwerpunkt zur Verfügung gestellt hat. Als Sohn eines Allgemeinmediziners mit Praxisschwerpunkt Kampo und mit japanischen Wurzeln versehen, recherchierte er über die möglichen Ursachen, warum Japan an der Spitze der Lebenserwartungsstatistik 
steht und einen hohen Anteil an Oldestold und Centenarians aufweist.

Gedanken zur Polypharmazie und Anleitungen zum Prozess des Deprescribing entstammen der Feder von Prof. Dr. Helmut Frohnhofen, Facharzt für innere Medizin und Geriatrie. Abgesehen davon, dass reduzierte Nieren- oder Leberfunktionen zu einer Kumulation von Medikamenten führen können, stören gewisse Substanzen die Regulationsfähigkeit des Organismus. Oftmals kann diese ohnehin durch das Altern per se reduziert sein, wie Hartmut Heine in der DZA schon dargestellt hat [2].

Im Beitrag „Alter und Geriatrie in der chinesischen Philosophie und TCM“ gibt Herr Dr. med. Fangju Kan einen Einblick in die Klassiker der chinesischen Medizin. „Es geht darum, in Würde (Konfuzianer) bzw. natürlich (Daoisten) zu altern“.

Den typischen Männerkrankheiten geht Prof. Dr. Alexander Meng auf den Grund und zeigt pragmatisch die wichtigsten Akupunkturpunkte auf. Und last, not least steht die Schönheit im Fokus des Überblicks über die kosmetische Akupunktur, den Frau Dr. Daniela Stockenhuber mit eindrucksvollen Fotos untermauert.

Vielleicht bietet dieses Schwerpunktthema nicht nur für Ihre Arbeit am und mit dem Patienten eine Hilfestellung, sondern motiviert Sie zu bewusstem Umgang mit den Fragen des eigenen Älterwerdens. Aktives, bewusstes, harmonisches Altern beginnt hier und jetzt.

\footnotetext{
Literatur

1. WHO https://apps.who.int/iris/bitstream/handle/10665/66941/WHO_NMH_NPH_01.2.pdf. Zugegriffen: 25. Aug. 2021

2. Heine H (2005) Alterungsprozesse an Akupunkturpunkten - Bedeutung für die Grundregulation. Dtsch Z Akupunkt 48(4):16-19
}

\section{Korrespondenzadresse}

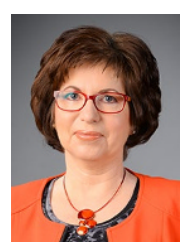

Dr. Michaela Bijak

Klinik Hietzing,

Österreichische Gesellschaft

für Akupunktur

Wolkersbergenstraße 1,

1130 Wien, Österreich

michaela.bijak@gesundheitsverbund.at

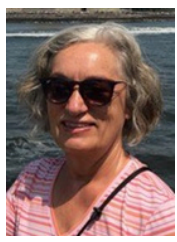

Dr. Cornelia Tauber-

Bachmann

Wasserloser Str. 32,

63755 Alzenau, Deutschland

praxis@tauber-medizin.de

Interessenkonflikt. M. Bijak und C. TauberBachmann geben an, dass kein Interessenkonflikt besteht.
In eigener Sache

\section{Schwerpunktübersicht Deutsche Zeitschrift für Akupunktur}

Die Deutsche Zeitschrift für Akupunktur bietet Ihnen umfassende und aktuelle Beiträge zu interessanten Themenschwerpunkten aus allen Bereichen der Akupunktur und verwandter Verfahren im Sinne einer integrativen Medizin.

\section{Überblick 2020 \\ - 1/20 Tuina \\ - 2/20 Das komplexe regionale Schmerzsyndrom \\ - 3/20 Ganzheit in der Medizin \\ - 4/20 Schlafstörungen}

\section{Überblick 2021}

- 1/21 Ohrakupunktur

- 2/21 Augenheilkunde

- 3/21 Elektrostimulationsakupunktur

- 4/21 Best Agers

Alle Inhalte der Zeitschrift finden Sie unter www.springermedizin.de/dza.

SpringerMedizin.de bietet Ihnen Zugang zu allen elektronisch verfügbaren Ausgaben Ihrer Zeitschrift - unabhängig davon, seit wann Sie die Zeitschrift abonniert haben.

Möchten Sie ein bereits erschienenes Heft nachbestellen? Einzelne Ausgaben können Sie direkt bei unserem Kundenservice zum Preis von je EUR 44,- zzgl. Versandkosten beziehen:

Springer Customer Service Center GmbH Kundenservice Zeitschriften

Tiergartenstr. 15, 69121 Heidelberg

Tel.: +49 $6221345-4303$

Fax: +49 6221345-4229

E-Mail: leserservice@springernature.com

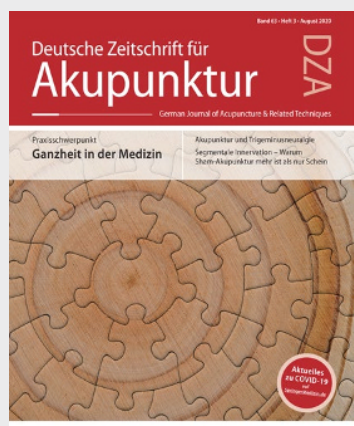

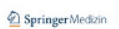

